Revue européenne des migrations internationales

vol. $21-n^{\circ} 1 \mid 2005$

Femmes, genre, migration et mobilités

\title{
La réussite paradoxale des bonnes espagnoles de
} Paris

Stratégies de mobilité sociale et trajectoires biographiques

Laura Oso Casas

\section{OpenEdition}

\section{Journals}

Édition électronique

URL : https://journals.openedition.org/remi/4222

DOI : $10.4000 /$ remi.4222

ISSN : $1777-5418$

\section{Éditeur}

Université de Poitiers

Édition imprimée

Date de publication : 29 mai 2005

Pagination : 107-129

ISBN : 2-911627-39-3

ISSN : 0765-0752

\section{Référence électronique}

Laura Oso Casas, «La réussite paradoxale des bonnes espagnoles de Paris », Revue européenne des migrations internationales [En ligne], vol. 21 - $\mathrm{n}^{\circ} 1$ | 2005, mis en ligne le 03 septembre 2008, consulté le 15 avril 2022. URL : http://journals.openedition.org/remi/4222 ; DOI : https://doi.org/10.4000/remi. 4222

Ce document a été généré automatiquement le 15 avril 2022

(c) Université de Poitiers 


\title{
La réussite paradoxale des bonnes espagnoles de Paris
}

Stratégies de mobilité sociale et trajectoires biographiques

\author{
Laura Oso Casas
}

« Il y a eu des gens qui ont su faire des économies. Ils vivaient en France comme durant la période de vaches maigres : le pot de lait condensé ils l'utilisaient comme tasse. Ils ne jetaient jamais le pain. Les femmes emportaient de l'eau de Javel, de la lessive, des maisons où elles travaillaient, parce que sinon, on ne gagne pas autant, juste en travaillant. Elles emportaient un torchon de la maison de la patronne, des petites choses. J'ai une amie qui invitait ses amis avec les meilleures boissons des patrons. Certaines choses sont justifiées. Celui qui vole un voleur, cent ans de pardon » (Marta et Dolores - FACEEF'1). «En Espagne les gens vivent mieux qu'ici. Ma sœur a un appartement, un magasin, elle a de l'argent et comme elle, beaucoup d'autres. Ils ne sont pas venus ici et ils vivent bien. Ici, tu peux beaucoup gagner, mais tu ne vis pas. Tu restes chez toi. Les gens s'enferment beaucoup. C'est à cause de la mentalité de l'émigré. On s'enferme parce qu'on veut épargner » (Pilar, qui migra seule, mariée, femme de ménage, $\mathrm{III}^{\mathrm{e}}$ arrondissement de Paris).

1 Depuis la fin des années quatre-vingt, l'Espagne est devenue un pays de destination pour de nombreuses femmes disposées à occuper les emplois socialement les plus dévalorisés. Femmes immigrées, employées domestiques, gardes d'enfants et de personnes âgées sont au centre des discours politique et scientifique dans l'Espagne 
d'aujourd'hui. Cette migration n'est pas sans rappeler l'aventure de toute une vie mettant en scène de jeunes Espagnoles arrivées dans les années 1960-1970 à Paris pour servir comme employées domestiques ou concierges. Dans ce texte, cherchant à sortir de l'oubli cette diaspora féminine ${ }^{2}$, nous nous intéressons à une migration qui, en son temps, a constitué une composante clé du développement économique espagnol et dont les protagonistes sont les Espagnoles qui ont quitté la péninsule pleines de courage et de rêves d'ascension sociale. Cependant, comme l'illustrent les citations ouvrant ce texte, certaines de ces femmes, dans la Ville de Lumière, ont à peine trouvé le miroitement de quelques francs laborieusement épargnés et l'âpreté d'une vie quotidienne assujettie à la stricte discipline du travail et de la frugalité. Les pages qui suivent invitent à un voyage dans le passé. Le caractère de flux migratoire ancien, éloigné dans le temps, permet d'évaluer les trajectoires biographiques des immigrées. Le vécu de nos interlocutrices qui appréhendent leur migration - comme une réussite ou un échec -, qui jugent leurs propres vies, est mis en lumière ${ }^{3}$.

2 L'article est centré sur les représentations, les stratégies et les trajectoires de mobilité sociale des femmes immigrées restées à Paris jusqu'à nos jours. Je présenterai le projet migratoire des femmes espagnoles ayant migré à Paris dans les années 1960-1970, centré sur la logique de l'épargne, du retour et de la projection de la mobilité sociale en Espagne. J'exposerai ensuite comment ce projet a configuré les stratégies et les trajectoires de mobilité sociale (professionnelles, résidentielles, d'épargne et de consommation) de ces femmes immigrées. Enfin, je présenterai l'évaluation par ces femmes de leur propre expérience migratoire. C'est ainsi que nous débouchons sur le paradoxe auquel sont confrontées les femmes espagnoles qui ont migré à Paris dans les années 1960-1970.

\section{La présence d'un courant migratoire féminin à caractère économique : les bonnes espagnoles à paris}

3 L'immigration espagnole est, avec l'italienne et la polonaise, l'une des plus anciennes de l'histoire migratoire française au $\mathrm{xx}^{\mathrm{e}}$ siècle. Déjà non négligeable avant les années trente (351 000 en 1931), la présence espagnole croît significativement à partir des années 1950 et plus particulièrement au cours des années soixante et soixante-dix, du fait de l'arrivée de la vague de migration économique ${ }^{4}$. Un demi-million de résidents espagnols (607 184) sont comptabilisés dans le recensement de 1968, l'immigration espagnole représentait à cette époque $23 \%$ du total des étrangers en France et constituait ainsi la première communauté d'immigrés. La présence des femmes, jusqu'au milieu des années soixante oscille entre 40 et $44 \%$ de l'ensemble de l'immigration espagnole en France. Ces premiers courants migratoires sont, comme le signale Gutiérrez (1984), fondamentalement familiaux. Depuis le milieu des années 1960, la présence féminine augmente progressivement et l'équilibre entre les deux sexes est atteint en 1999 (49,8\%). 
Tableau 1 : Espagnols en France par sexe 1911-1999

\begin{tabular}{|r|c|c|c|c|c|}
\hline & Ensemble & Femmes & \% Femmes & Hommes & $\%$ Hommes \\
\hline 1911 & 105760 & 44905 & 42,5 & 60855 & 57,5 \\
\hline 1921 & 254980 & 102868 & 40,3 & 152112 & 59,7 \\
\hline 1926 & 322590 & 139036 & 43,1 & 183554 & 56,9 \\
\hline 1931 & 351864 & 151728 & 43,1 & 200136 & 56,9 \\
\hline 1936 & 253599 & 113075 & 44,6 & 140524 & 55,4 \\
\hline 1946 & 302201 & 119947 & 39,7 & 182254 & 60,3 \\
\hline 1954 & 288923 & 121359 & 42,0 & 167564 & 58,0 \\
\hline 1962 & 441658 & 194605 & 44,1 & 247053 & 55,9 \\
\hline 1968 & 607184 & 284276 & 46,8 & 322908 & 53,2 \\
\hline 1975 & 497480 & 235115 & 47,3 & 262365 & 52,7 \\
\hline 1982 & 327156 & 152080 & 46,5 & 175076 & 53,5 \\
\hline 1990 & 216047 & 103700 & 48,0 & 112347 & 52,0 \\
\hline 1999 & 161762 & 80609 & 49,8 & 81153 & 50,2 \\
\hline
\end{tabular}

Source : Élaboration par l'auteur à partir des Recensements de la population en France, INSEE.

4 Selon Gutiérrez, se développe dans les années soixante un flux migratoire composé de femmes seules, principalement célibataires, qui se dirigeaient massivement vers Paris et sa région, en quête d'un emploi dans le service domestique, l'immigration espagnole revêtant un caractère plus familial dans le reste du territoire (Gutiérrez, 1984) ${ }^{5}$. Comme le note cet auteur, en 1931, la proportion d'Espagnoles employées dans la catégorie "personnel de service » en France était de $14 \%$, tandis qu'en 1968 elle était passée à $53 \%$ (38 860 femmes). Ce secteur d'activité en 1968 regroupait $72 \%$ des Espagnoles employées dans la Région parisienne et 79 \% à Paris. «En 1975 la Région parisienne concentrait près de la moitié de l'ensemble des Espagnoles actives en France. La même année, les deux tiers des Espagnoles qui travaillaient dans le secteur des services vivaient dans la Région parisienne» (Gutiérrez, 1984: 620). Ainsi, la figure de la «bonne espagnole » se consolide dans la société française ${ }^{6}$. Nous sommes, donc, face à une migration de femmes correspondant peu au stéréotype de l'immigrante inactive, venue dans le cadre du regroupement familial et dépendante de l'homme migrant. Ce courant migratoire de femmes s'est développé au même moment que celui des hommes espagnols qui s'intégraient principalement dans l'industrie automobile ou dans le bâtiment.

Trente ans se sont écoulés depuis que ces femmes sont arrivées en France. Combien de femmes espagnoles vivent-elles encore à Paris et dans la Région parisienne ? En 1990, il y avait 30108 Espagnoles en Île-de-France, ce qui représentait 50,5\% de l'ensemble des Espagnols de la région, 12168 résidaient dans la Ville de Paris (54,3\% de l'ensemble des Espagnols à Paris). À la même époque, dans l'ensemble du pays 12628 Espagnoles actives travaillaient comme personnel des services directs aux particuliers $(35,5 \%$ de l'ensemble des actives espagnoles en France). Les données les plus récentes, issues du recensement de 1999 mettent en relief l'existence de 36766 Espagnoles en Île-deFrance ( $57 \%$ de l'ensemble des Espagnols dans la région). La forte féminisation dans les années quatre-vingt-dix (plus de $7 \%$ en 9 ans) procède du vieillissement de cette population.

Les données qualitatives que nous allons présenter sont issues d'un travail de terrain mené à Paris en 1998-1999, dans les $\mathrm{XVI}^{\mathrm{e}}$ et $\mathrm{XI}^{\mathrm{e}}$ arrondissements. Le XVI ${ }^{\mathrm{e}}$ arrondissement de Paris est l'une des zones les plus huppées de la capitale française. 
Ici, les immigrantes espagnoles étaient employées comme concierges ou domestiques, résidant, la plupart du temps, sur leur lieu de travail (chambre de service, loge de concierge). Cependant, une autre réalité est celle de l'immigration qui s'était installée dans les quartiers ouvriers comme le $\mathrm{XI}^{\mathrm{e}}$ arrondissement, où les modalités d'emploi (heures de ménage, ramassage des poubelles, nettoyage des bureaux, ateliers de couture) et de résidence (location, chambre d'hôtel - hôtel meublé, HLM, loge de concierge) variaient dans une certaine mesure ${ }^{7}$. Au total, soixante-six personnes ont été contactées et interviewées formellement, dont 49 femmes immigrées ${ }^{8}$ (voir tableau 2).

Tableau 2 : Personnes contactées pendant le travail de terrain réalisé à Paris, 1998-1999

\begin{tabular}{|l|c|c|c|}
\hline & Hommes & Femmes & Ensemble \\
\hline Immigrés espagnols à Paris & 7 & 49 & 56 \\
\hline Informateurs clé & 2 & 8 & 10 \\
\hline Ensemble & 9 & 57 & 66 \\
\hline
\end{tabular}

7 Parmi les 49 femmes espagnoles interviewées, la majorité (30) arrivèrent à Paris dans le cadre d'une migration qu'elles ont organisée et initiée. Il s'agissait d'une migration à caractère économique, non dépendante de l'homme, composée de femmes qui s'inséraient comme bonnes à tout faire, notamment dans des quartiers «bourgeois » comme le $\mathrm{XVI}^{\mathrm{e}}$ arrondissement. La plupart d'entre elles le firent comme célibataires, une minorité firent le voyage comme veuves, séparées ou divorcées. La deuxième modalité la plus fréquente que nous avons rencontrée parmi les femmes interviewées est celle de la migration en couple : l'homme et la femme migrent ensemble en France (neuf cas). Certaines de nos interviewées vinrent dans le cadre du regroupement familial, le mari étant le pionnier de la migration (sept cas). Enfin, trois de nos interlocutrices, pionnières de la migration, ont été les premières à partir du pays et à faire venir ultérieurement leur mari, après avoir trouvé un logement et un travail, comme bonnes à tout faire.

Tableau 3 : Personnes contactées pendant le travail de terrain réalisé à Paris, selon le type de migration, 1998-1999

\begin{tabular}{|l|c|}
\hline Migration de femmes seules (célibataires, veuves, séparées/divorcées) & 30 \\
\hline Migration en couple & 9 \\
\hline Femmes pionnières de la migration familiale & 3 \\
\hline Femmes arrivées dans le cadre du regroupement familial & 7 \\
\hline Ensemble & 49 \\
\hline
\end{tabular}

\section{Représentations, stratégies et trajectoires de mobilité sociale des femmes immigrées : une approche théorique}

8 La théorie de Blau et Duncan (1967), qui, selon Cuin (1993), a été dès son apparition dominante dans le cadre de la sociologie de la mobilité sociale aux États-Unis, se base sur les valeurs de l'universalisme et de l'achievement, comme principes orientant les actions individuelles, afin d'expliquer les processus de mobilité sociale. Selon Cuin, le 
système idéologique nord-américain a développé la croyance dans l'égalité des chances, ce qui lui permet de légitimer les inégalités existantes dans la structure sociale, cellesci n'ayant pas une base structurelle, déterminée socialement, mais individuelle (l'«American Creed »). De ce point de vue, les inégalités sont principalement basées sur les compétences et les qualités personnelles. Cependant, l'égalité des chances n'est pas suffisante, et le système idéologique a besoin d'une croyance fondamentale pour que la liberté individuelle et l'égalité sociale puissent se conjuguer: la fluidité ou la libre circulation, sans barrières, des individus dans la structure sociale, la nécessité d'une société ouverte, de telle sorte que n'importe quel individu puisse se déplacer dans la structure de classes, lui conférant la possibilité de réussir. Ainsi, la mobilité sociale estelle indispensable au maintien du système idéologique nord-américain (Cuin, 1993).

Loin de la vision centrée sur l'individu rationnel, qui calcule sa mobilité sociale, dans le cadre d'une société ouverte (Blau et Duncan, 1967), nous considérons que la mobilité sociale des migrants est conditionnée par des déterminants structuraux. Cependant, notre approche théorique n'offre pas non plus une vision balistique de la mobilité sociale, de sorte que nous y incorporons l'action de l'acteur social. Ainsi, notre modèle d'interprétation se base fondamentalement sur l'articulation de trois notions: représentations, stratégies, et trajectoires de mobilité sociale.

En premier lieu, la notion de représentations nous permet de poser que l'individu se représente soi-même, en relation avec d'autres sujets, imagine sa position sociale et projette sa mobilité sociale et celle des siens. La mobilité sociale se voit, donc, déterminée par les représentations que les individus s'en font. Les images autour des possibilités et des mécanismes d'ascension sociale contribuent à la construction de la réalité sociale. L'idée de programmer la migration et d'associer le déplacement géographique à la mobilité sociale est souvent la base sur laquelle les individus s'appuient pour décider de leur processus migratoire. Les migrants potentiels évaluent les bénéfices de la migration en fonction des représentations du pays de destination et des expectatives de mobilité sociale.

11 En ce qui concerne la notion de stratégies, il faut signaler que : "Si l'on dit d'un individu qu'il a un comportement stratégique, cela veut dire non seulement que sa conduite est rationnelle, qu'il effectue ses choix d'action en fonction de la perception de ses intérêts et des risques que certains choix peuvent lui faire courir, mais aussi qu'il se voit dans le futur, préférant un moindre gain aujourd'hui, pour un avantage plus stable ou plus important demain » (Gesle et al., $1994: 356$ ).

12 La notion de trajectoire permet d'étudier la relation entre mobilité géographique et mobilité sociale. La trajectoire sociale serait l'ensemble des positions sociales successives qu'occupe un individu au cours de son existence. Par trajectoire de mobilité sociale, nous faisons référence aux itinéraires personnels ou collectifs des individus en fonction d'un ou de plusieurs espaces sociaux donnés.

13 En résumé, les individus se représentent la mobilité sociale, ils mettent en pratique une série de stratégies afin de réaliser leur désir d'ascension sociale. Ces stratégies et ces représentations vont se combiner avec celles d'autres acteurs sociaux : parents, voisins, relations amicales, employeurs, représentants politiques, etc. Les stratégies de mobilité sociale ne sont pas seulement individuelles, mais aussi familiales. Ce que nous cherchons à démontrer dans cet article c'est que les représentations et les stratégies de mobilité sociale, les projets et les buts d'ascension sociale, tracés par les femmes immigrées, peuvent finalement déboucher, du fait du poids des déterminants 
structurels, de la réorientation des stratégies initiales, de la position même de l'individu dans le cycle migratoire et de vie, sur des trajectoires de mobilité sociale distinctes et même opposées à celles dont les femmes immigrées avaient rêvé. Ainsi, les représentations construites de la mobilité sociale et les trajectoires de mobilité sociale ne vont pas toujours de paires, elles peuvent même entrer en contradiction et en conflit; des conflits d'intérêts entre les membres du foyer et d'autres acteurs sociaux. La mobilité sociale des femmes immigrées peut aussi se voir contrainte par des facteurs d'ordre structurel (situations du marché du travail, politique migratoire, etc.).

Notre analyse de la problématique migration et mobilité sociale implique de prendre en compte au moins deux espaces sociaux, celui que quitte le migrant dans la communauté d'origine et l'espace social d'accueil ; ces espaces sont gérés par des structures et des hiérarchies sociales distinctes, la position des migrants pouvant varier dans chacun d'entre eux. Ainsi, une personne peut connaître une mobilité sociale descendante en migrant et, cependant, améliorer son statut dans l'espace social d'origine (Oso, 2002).

\section{Le projet d'épargne, de retour et la projection de la mobilité sociale en espagne}

Dans le premier tiers $\mathrm{du} \mathrm{xx}^{\mathrm{e}}$ siècle, on assiste à une tentative de modernisation capitaliste en Espagne avec le développement d'un premier "modèle de consommation ». L'apparition de la publicité de produits de consommation ne s'adresse qu'aux classes argentées et à une petite bourgeoisie urbaine alors que les classes défavorisées sont victimes d'une "surexploitation extensive " (Alonso et Conde, 1994 : 65). Une idéologie destinée à la masse ouvrière promeut la discipline du travail et de l'épargne, de telle sorte qu'un «discours moralisateur tente de présenter l'emploi comme une vertu morale par excellence » (Alonso et Conde, 1994 : 66). Le texte écrit sur les premiers livrets d'épargne en Espagne reflète parfaitement ce discours de la thésaurisation comme un bien moral pour les travailleurs :

«Comment peut-on épargner? En suivant à la lettre les règles suivantes :

Dépense toujours moins que ce que tu gagnes.

Évite les dettes.

Paie comptant.

Ne sors pas de la strate sociale où tu vis.

Si tu n'es pas riche, n'essaie pas de le paraître.

Vis en accord avec la classe sociale à laquelle tu appartiens.

N'oublie jamais les petits détails. Ce sont eux qui te permettront d'épargner ${ }^{9}$.

La tentative de modernisation fut freinée par la Guerre Civile espagnole et par l'instauration du franquisme autarcique. Le bref modèle de "consommation d'élite " qui germa au début du siècle en Espagne sombrera dans une longue léthargie durant les vingt premières années de la dictature franquiste. Jusqu'aux années 1950, le niveau de production industrielle atteint antérieurement à la guerre ne sera pas égalé. S'amorce, à partir de ce moment, une période de reprise économique avec l'appui des États-Unis. Entre 1947 et 1956, on considère que l'Espagne a mis fin à son isolement international, elle sera intégrée au système économique mondial à la fin des années $1950^{10}$. En 1958, l'OCDE recommanda au gouvernement espagnol la mise en place d'un plan de stabilisation économique qui sera approuvé l'année suivante. De 1960 à 1973, survient une période de croissance économique, appelée «el desarrollismo español ${ }^{11}$, qui se fonde principalement sur le passage d'une économie basée sur l'agriculture à une 
économie fondée sur l'industrie, et dans une moindre mesure sur le développement des services, sur la croissance du pouvoir d'achat ainsi que sur l'acquisition de technologies avancées. Afin d'amortir les coûts d'importation de biens d'équipement, le gouvernement s'est appuyé sur les entrées de devises liées au tourisme et sur les transferts monétaires des émigrés (Angoustures, 1995). L'émigration vers les pays du Nord de l'Europe permettra d'éliminer un important excédent de main-d'œuvre sur le marché du travail espagnol. Pour assurer l'envoi de mandats, le gouvernement a développé une politique d'ouverture de comptes bancaires et a promu une approche transitoire et fonctionnelle de l'émigration que les émigrés ont intériorisée, jusqu'à devenir une forme d'ethos. Dans une perspective paternaliste, l'émigration fut considérée par le régime franquiste comme «une fatalité bienfaitrice». Vont se répandre des discours patriotiques manifestant le rejet de «l'étranger", consolidant les signes d'appartenance à l'identité espagnole et développant l'idée que l'émigration ne saurait être que brève; la figure de l'émigré devient celle du sacrifié. L'administration espagnole va mettre en place un ensemble de structures chargées d'encadrer les émigrés dans les pays d'accueil qui, tout en s'efforçant de maintenir l'identité culturelle espagnole, va chercher à les préserver et à les protéger de l'intégration dans les pays concernés (Aragón, 1986).

Au début des années 1960, avec le développement économique, les classes moyennes commencent à alimenter le modèle de la société de consommation de masse alors que l'Espagne sort à peine de la pénurie de l'après-guerre et de l'autarcie (Alonso et Conde, 1994). Contrairement à cette pratique de consommation dorénavant généralisée en Espagne, les personnes que nous avons interviewées ont développé une stratégie d'épargne assise sur un projet migratoire à court terme orienté vers le retour et la mobilité sociale au pays d'origine. Elles ont intégré, de façon fonctionnelle, l'ethos de la culture ouvrière du labeur, de l'austérité et de l'épargne prévalant en Espagne avant les années 1960. L'objectif des migrants était de travailler le plus possible, en maximisant leurs gains et leur épargne. La migration était un "sacrifice» temporaire visant à obtenir une récompense: la mobilité sociale en Espagne. L'ascension sociale fut projetée dans l'espace social d'origine (en Espagne) et non d'accueil (en France); les migrants différant ainsi leur consommation jusqu'au retour. Cette représentation de la migration, alimentée par le franquisme, a orienté ultérieurement les trajectoires professionnelles et résidentielles des migrants.

\section{Trajectoires professionnelles et résidentielles}

Dans les années 1960-1970, une forte proportion d'Espagnoles qui avaient émigré à Paris ont travaillé comme bonnes à tout faire, particulièrement dans le $\mathrm{XVI}^{\mathrm{e}}$ arrondissement. Cette stratégie professionnelle permettait de couvrir les frais de manutention et de logement, notamment parce que la "chambre de bonne", indépendante de l'appartement des " patrons ", avec sanitaires à l'extérieur, a permis, dans certains cas, de regrouper la famille et d'alimenter les réseaux migratoires. Certaines femmes ont migré en premier et ont fait ensuite venir leurs maris, leurs fiancés, ou leurs amis, avec lesquels elles pouvaient partager, temporairement ou à plus long terme, «la » chambre de bonne. L'emploi comme «bonne à tout faire» rendait possible l'épargne monétaire, bien que les conditions de travail et de vie fussent très astreignantes pour les migrantes cantonnées dans un espace de quelques mètres carrés 
et soumises à une domination paternaliste caractéristique du service domestique à demeure.

Les femmes qui sont parties avec leurs compagnons ou leurs époux adoptèrent des stratégies professionnelles et résidentielles un peu distinctes de celles des femmes seules du XVI ${ }^{\mathrm{e}}$ arrondissement. Elles s'embauchaient comme employées domestiques à l'heure ou comme couturières. En général elles cumulaient les emplois : nettoyage de bureaux de six à neuf, nettoyage à l'heure dans différentes maisons et travaux de couture. Les hommes travaillaient le plus souvent dans le bâtiment ou dans l'industrie automobile. La stratégie résidentielle consistait à trouver des chambres de service (louées ou occupées en échange d'heures de nettoyage) ou des chambres d'hôtels. Dans le $\mathrm{XI}^{\mathrm{e}}$ arrondissement de Paris, on trouvait de nombreux hôtels remplis d'immigrés espagnols : de petites chambres, avec sanitaires à l'extérieur et sans eau.

Compte tenu de la composition démographique du courant migratoire des années 1960 - beaucoup de femmes (les bonnes) et d'hommes (les ouvriers du bâtiment et de l'industrie automobile) qui migrèrent étaient célibataires et seuls -, de multiples unions furent contractées entre immigrés. L'église de la Pompe (Paris $\left.16^{\circ}\right)$, les salles de bals de Wagram et du Bataclan, dans les premières années de la migration, ainsi que les associations espagnoles plus tard, ont constitué des centres de réunion où les hommes et les femmes liaient connaissance. Les femmes qui se marient délaissaient généralement le statut de bonnes à tout faire, pour celui de femmes de ménage et se consacrer au nettoyage des bureaux. Certaines des célibataires du XVI ${ }^{\mathrm{e}}$ arrondissement sont parties vivre, après leur mariage, avec leurs maris dans des quartiers populaires.

21 L'arrivée des enfants retarda le retour et réorienta les projets initiaux. Les bénéficiaires de l'ascension sociale programmée étaient maintenant les enfants. Le passage du couple à la famille nucléaire entraîna également une nouvelle formulation des stratégies professionnelles et résidentielles. Pour la femme, le travail comme employée domestique à demeure, ainsi que les heures de nettoyage dans des maisons et des bureaux, cessent d'être une option privilégiée alors qu'il faut élever les enfants. De façon générale la stratégie professionnelle et résidentielle adoptée fut l'emploi comme concierge. La loge de concierge permettait de disposer d'un logement gratuit pour l'ensemble de la famille. La capacité d'épargne du couple migrant se voyait, en outre, peu entamée, dès lors que la femme continuait à percevoir un salaire tout en s'occupant de sa famille. La loge de concierge rendait même possible l'apport de gains supplémentaires, pas déclarés, comme la couture, le lavage et repassage des vêtements des voisins, les pourboires, les heures de ménage. Et le couple continuait à organiser sa vie dans une logique d'épargne en acceptant les sacrifices que supposait la migration: nombre de loges de concierges, par exemple, n'avaient qu'une seule pièce que devaient se partager parents et enfants.

Elena a migré à Paris dans le cadre du regroupement familial. Son mari représente parfaitement la figure du migrant tenaillé par le projet d'épargne et du retour. Le couple commença son périple migratoire dans une petite chambre louée dans le $\mathrm{XX}^{\mathrm{e}}$ arrondissement de Paris. La stratégie du cumul d'emplois fut mise en pratique par notre interlocutrice au début du cycle migratoire : couture, heures de ménage, etc. À l'arrivée des enfants, le couple opte pour une loge de concierge :

«Lorsque je suis arrivée, mon mari avait une chambre, où il y avait un lit et une armoire. De l'autre côté de la chambre, il y avait une petite plaque, une table et une chaise. Lorsque je suis tombée enceinte, nous avons pris une loge. C'était une 
chambre et, sous l'escalier, il y avait une cuisine très petite. Nous dormions sur un canapé-lit la nuit et le jour c'était le salon. C'était pas bien grand, mais on rentrait dedans. Une table, trois chaises, une armoire et une télé. Nous n'avions pas de salle de bain, nous devions nous laver dans une cuvette et c'était comme ça. Moi, je lavais mes enfants dans la cuvette le soir et le matin, je les lavais aussi avant d'aller à l'école. Le W.C. était à l'extérieur et les affaires de toilette nous devions les laisser dehors parce qu'il n'y avait pas de place à l'intérieur » (Elena, venue rejoindre son époux, $\mathrm{XI}^{\mathrm{e}}$ arrondissement de Paris).

\section{Les stratégies de consommation et d'épargne ${ }^{12}$}

Les comptes d'épargne promus par le gouvernement espagnol pour capter les transferts des migrants constituent un élément clé qui a permis d'alimenter les stratégies de thésaurisation. Ainsi, les Espagnols envoyaient-ils tous les mois la majeure partie de leur salaire au pays afin de profiter des intérêts spéciaux appliqués à leurs comptes. Pour pouvoir impulser le projet migratoire d'épargne et de retour, il était nécessaire de combiner les stratégies professionnelles et résidentielles, qui facilitaient l'accumulation monétaire (employée comme bonne à tout faire, cumul d'emplois, loge de concierge) avec des pratiques de consommation parcimonieuses qui supposaient de réduire les dépenses au strict minimum afin d'envoyer le maximum d'argent en Espagne.

« Nous ne sortions jamais. Nous ne faisions rien qui pouvait nous coûter de l'argent, parce que tout ce que tu dépensais, tu ne pouvais pas l'envoyer là-bas. La mentalité était d'envoyer le maximum au plus tôt. Maintenant, je pense que j'aurais pu en profiter plus. Nous, le premier argent que nous avons gagné a été pour s'acheter un appartement au village. L'argent, nous l'envoyions sur un compte d'épargne là-bas. Ce que tu réussissais à économiser, tu l'envoyais là-bas, on vivait avec un salaire et tu envoyais l'autre là-bas, de cette façon, il restait quelque chose au cas où tu aurais eu un problème » (Luisa, pionnière de la migration familiale, $\mathrm{XVI}^{\mathrm{e}}$ arrondissement de Paris).

L'accumulation monétaire, qui parfois frôlait l'avarice, conduisait certaines femmes titulaires de comptes courants en Espagne, à faire les poubelles. Le travail de certaines Espagnoles, en contact avec les déchets domestiques, leur a permis de recycler et de récupérer des objets.

«Moi, j'en connais une qui a beaucoup d'argent et un jour, je suis rentrée dans sa salle de bains. Elle vit dans une loge de concierge et je suis restée stupéfaite. Avec tant d'appartements et tant d'argent en Espagne, elle avait accroché un miroir dans la salle de bains, qu'elle avait récupéré dans la poubelle ! Ces gens, ils ne vivent pas. Ils profitent en accumulant, c'est de l'avarice. Moi, un miroir comme ça je le jette. Ils vivent pour accumuler et gagner. Tout pour la retraite, même s'ils ne savent pas s'ils y arriveront " (Entretien avec un groupe de quatre femmes espagnoles, XVI ${ }^{\mathrm{e}}$ arrondissement de Paris).

D'après nos interlocutrices, certaines familles contrôlaient au plus près leurs dépenses alimentaires. C'est le cas de la famille du «pollastro " («poulet» avec une connotation péjorative, mot usité pour décrire les immigrés qui mangeaient du poulet toute la semaine) ou des immigrants "pataconeros» («des pommes de terre», un terme tout aussi péjoratif pour décrire ceux qui se nourrissaient de pommes de terre). Les pratiques d'épargne rapportées font écho à celles en cours dans la société espagnole durant les années d'après-guerre : utiliser un pot de lait condensé comme tasse, garder le pain dur, etc. D'autres pratiques consistaient à prendre du savon et des torchons 
dans les maisons des patrons, des petits morceaux de tissu, un peu de sucre, de sel, quelques cigarettes... ou encore à porter, cas plus rare, des vêtements et des chaussures hérités des patronnes. Enfin nos interlocutrices ont mentionné des cas extrêmes, ceux de familles faisant travailler leurs enfants dans la couture pour augmenter les revenus du foyer.

Certaines des immigrées connues de nos interlocutrices allaient même jusqu'à masquer leur « réelle condition économique » en se présentant avec des tenues vestimentaires modestes afin d'obtenir quelques avantages venant s'ajouter à leurs rémunérations : pourboires, cadeaux des patronnes et des habitants de l'immeuble (vêtements, chaussures, objets, etc. $)^{13}$.

« Nous en connaissons une qui se promenait avec des chaussures abîmées et avec les chaussettes du mari et qui nous a dit: "Je suis mal habillée chez les patrons, parce qu'ainsi ils me donnent de tout. Je ne m'habille pas bien, parce que sinon, ils penseraient que j'ai de l'argent. Je veux éveiller leur compassion". Les patronnes ne savent pas ce qu'elles ont. Elles sont mal habillées pour qu'on leur donne quelque chose » (Piedad et Maruja, mariées, pluriactivité, $\mathrm{XI}^{\mathrm{e}}$ arrondissement de Paris).

Le projet d'accumulation monétaire a conduit à un repli sur la famille pour celles qui étaient mariées par exemple, la journée se résumant au travail. Il était alors inconcevable d'établir des relations sociales sans dépenser en loisirs et s'aménager du temps libre (aller au restaurant, au café, etc.). De même, les invitations à dîner chez des amis ou connaissances devaient être rendues, sources également de dépenses. En outre, la socialisation en Espagne et la logique de l'épargne ne prédisposaient pas à la consommation. Et l'isolement n'a pas contribué à altérer leur mentalité malgré le temps. Rivées à leur projet d'accumulation monétaire, certaines employées domestiques à demeure ont sombré dans la démence, confinées dans un espace réduit (leur chambre de bonne), ayant fait le sacrifice de tant d'années de pauvreté quotidienne pour atteindre la retraite et disposer de quelques millions de pesetas en banque.

« Nous n'allions jamais au restaurant. Ici, on n'achetait rien, nous n'avions pas le droit de dépenser un franc. Nous dépensions seulement pour l'alimentation, et dans les études de nos filles. Je n'avais pas le droit de m'acheter des vêtements. Tout était pour l'Espagne. On envoyait tout d'Espagne, ici nous n'achetions rien. Tout ce que nous avions gagné, on l'envoyait en Espagne. Nous achetions tout en Espagne: vêtements, charcuteries, chaussures... Nous n'avons pas dépensé un centime en France. Moi je n'ai pas vécu ici, j'ai vécu en Espagne, si mon mari voit que j'achète des meubles, il a une crise cardiaque, il me dit: "ça y est, tu dépenses encore notre argent". Mon mari regardait toujours les prix, l'épargne et tout ça et il fait vingt fois le tour du marché avant d'acheter quoi que ce soit. Nous venions ici uniquement pour travailler et économiser, nous n'avions pas le droit de manger des langoustines, ni d'aller au cinéma. Nous ne sortions jamais, nous étions seuls, très seuls. Mon mari, il faisait juste manger, travailler et dormir» (Elena venue rejoindre son époux, $\mathrm{XI}^{\mathrm{e}}$ arrondissement de Paris).

" Il y a eu une fille qui habitait dans le XVI et qui s'est jetée à la Seine. On a trouvé dans sa chambre une grosse somme d'argent et des livrets de caisse d'épargne avec un nombre de millions impressionnants. Elle vivait dans une chambrette. Il y a beaucoup de dépressions parmi les femmes âgées " (Carmen, qui migra à Paris seule).

28 La réussite sociale se mesure au succès économique atteint dans le pays d'origine. À Paris, on vit de façon austère, on n'a pas besoin de paraitre face à ses compatriotes. Dans la capitale, l'ostentation se manifeste par le biais des photos de la maison de campagne, de l'appartement, etc. De plus, la distance géographique permet de cacher 
aux familles en Espagne les véritables conditions de la vie quotidienne des migrants, la position peu élevée dans la structure sociale : bonne, femme de ménage, concierge ${ }^{14}$. En Espagne, toutes les dépenses étaient justifiées et les immigrées différaient leurs achats : vêtements, chaussures, loisirs etc. Les immigrés profitaient de leurs propriétés pendant les vacances, la maison symbolisant la «réussite» sociale au regard de la société d'origine :

«Certaines années, nous dépensions un million de pesetas en vacances (6000 euros). Parce que nous passions la journée au bar ; nous prenions le petit-déjeuner au bar, l'apéritif au bar, nous dînions au bar. Nous allions à la plage toute la journée, ensuite ma mère venait puis ma sœur et ensuite d'autres membres de la famille, nous étions dix ou douze pour manger et c'était moi qui payais tout. Nous allions au parc aquatique. Lorsque nous arrivions en Espagne, tout le monde nous regardait comme des riches. On te voyait prendre le petit-déjeuner au bar, on disait: "bien sûr, comme ils sont riches". Cette phrase est la plus utilisée pour qualifier les émigrants. Moi je ne prenais pas ça mal, parce que je pensais: "si je suis riche ou nouveau riche et si j'ai de l'argent, c'est parce que j'ai travaillé comme une mule". Nous arrivions avec de belles voitures. Là-bas nous avions le droit de dépenser ce que nous ne pouvions pas dépenser ici. La mentalité de mon mari était comme ça. Il trouvait toujours des problèmes pour acheter ici, si mes filles avaient besoin de vêtements ou de chaussures, il disait : "que c'est cher ! Attendons de retourner en Espagne pour dépenser". En Espagne, mon mari voulait toujours aller faire les magasins. On achetait tout en Espagne. Il disait : “j'ai besoin de pantalons, lorsque nous irons en Espagne je m'en achèterai une paire" " (Elena, venue rejoindre son époux, $\mathrm{XI}^{e}$ arrondissement de Paris).

Au début du cycle migratoire, le modus vivendi, centré sur le travail, l'épargne et la consommation minimale s'est généralisé. La plupart des femmes interviewées indiquent que, pendant les premières années de la migration, la stratégie migratoire exigea de plus amples sacrifices en termes de confort résidentiel et de dépenses en loisirs et en temps libre, qui étaient limitées. Actuellement, dans une position avancée du cycle migratoire, les stratégies initiales des migrants se sont transformées. L'idée de l'épargne et du retour est encore présente chez certains migrants tandis que pour d'autres, elle n'est plus aussi marquée et s'efface même avec le temps. Bien que la plupart des immigrantes espagnoles interviewées aient choisi d'investir leur argent en Espagne, principalement dans l'achat d'une ou plusieurs propriétés, d'autres décidèrent d'acheter un logement à Paris, ce qui témoigne d'un projet migratoire restructuré, qui n'était plus autant orienté vers la société d'origine, mais vers l'amélioration des conditions de vie à Paris. Cependant, parmi ceux qui ont décidé d'investir dans un logement à Paris, l'illusion ultime du retour en Espagne ne s'est pas nécessairement estompée. La capacité d'épargne a également été réduite pour les migrants qui ont décidé d'investir dans l'éducation de leurs enfants.

«Moi, j'ai acheté un appartement ici parce que je me disais: "ici, je vis pendant onze mois" et je pensais que c'était normal de vivre mieux pendant onze mois comme une personne et que mes enfants aient une vie normale, au lieu de vivre un mois seulement en Espagne et vivre dans un château pendant un mois. Ensuite, j'ai également acheté en Espagne, mais j'ai d'abord acheté ici [...]. La fortune nous l'avons déjà. Moi, je suis contente de ce que j'ai fait. Maintenant, il faut vivre au jour le jour, le mieux possible, parce que pour le peu qu'il nous reste à vivre, il faut en profiter, avoir une vie tranquille " (Inés, qui migra seule, mariée ultérieurement, femme de ménage).

«Maintenant les gens dépensent un peu plus. Les gens se sont rendus compte que la vie filait. Mais, il en reste encore pas mal qui ne sortent pas. Maintenant, nous avons des sorties, nous avons des activités, nous allons au cinéma, on va voir des 
spectacles de flamenco. Les gens sortent plus. Avant, c'était très difficile qu'ils dépensent quoi que ce soit, parce que les gens épargnaient et envoyaient tout, cela se fait de moins en moins " (Encarna, qui migra avec son époux, concierge, $\mathrm{XI}^{\mathrm{e}}$ arrondissement de Paris).

\section{Le paradoxe de l'immigration espagnole à paris} décision du retour se prenait lorsque les enfants arrivaient à l'âge scolaire. Ceux qui ont opté pour la scolarisation en France sont restés. À partir du milieu des années 1970, la suspension du recrutement officiel de main-d'œuvre étrangère, la fin de la dictature franquiste et la croissance économique du pays ont incité aux retours ${ }^{15}$, mais dans une proportion moindre que ce qui avait été programmé par les migrants : les uns retenus par leurs descendants, les autres par la crainte de ne pas trouver de travail en Espagne. Nos interlocuteurs ne se sont pas décidés à franchir le pas, et ont continué à prolonger leur projet migratoire. Les enfants terminèrent l'école, mais de nouveaux déterminants empêchaient le retour : l'arrivée des petits-enfants, l'attente de la retraite, etc. À quoi l'on peut ajouter la peur des difficultés d'intégration dans la société espagnole: le retour ressemblait à une autre migration qui supposait une nouvelle adaptation, difficile à surmonter pour une population d'âge avancé.

La dynamique de la migration échappa au contrôle des migrants : ces derniers se trouvèrent happés par un projet familial. Entre-temps la situation en Espagne avait considérablement changé, ce pays connaissait un développement économique et oubliait ses émigrés. Le contexte de la migration s'était transformé, bien que la mentalité de certains n'ait pas nécessairement changé, de sorte que quelques-uns ont continué à vivre sous le principe du sacrifice à Paris en projetant leur mobilité sociale dans le pays d'origine. D'autres, comme nous l'avons déjà signalé, s'adaptèrent un peu plus au contexte d'accueil et restructurèrent leur stratégie migratoire initiale. Ils commencèrent à projeter leur avenir non seulement en Espagne, mais aussi en France. La trajectoire sociale des Espagnoles qui résident aujourd'hui à Paris signifie-t-elle un échec des projets et des stratégies migratoires d'ascension sociale? Comment nos interlocutrices évaluent-elles leur expérience migratoire?

Dans la société d'accueil, les conditions de travail s'améliorèrent au fur et à mesure qu'avançait le cycle migratoire. Le travail le plus précaire, comme bonne à demeure, fut laissé de côté et les Espagnoles s'employèrent comme concierges, en nettoyant à l'heure les bureaux, les maisons ou comme assistantes maternelles, de sorte qu'aujourd'hui les Espagnoles figurent parmi les femmes immigrées qui jouissent des meilleures conditions légales et salariales de travail à Paris. Cependant, malgré ces progrès, les travaux de nettoyage et de soins personnels ne furent pas, pour beaucoup d'immigrées, abandonnés au profit d'autres emplois moins dévalorisés socialement. La mobilité professionnelle fut donc limitée. De plus, à Paris, la mobilité résidentielle a été bloquée par le projet du retour. La plupart des investissements ont été réalisés dans la société d'origine, ce qui implique, au-delà de la loge de concierge et des HLM, un blocage des mécanismes d'amélioration des conditions de logement. D'autre part, l'ethos de l'épargne a limité, pour beaucoup, les pratiques de consommation, de loisirs et de temps libre, fermant la porte à une amélioration de la qualité de vie à Paris. De même, l'accumulation économique comme principal objectif migratoire a eu un effet pervers sur le capital social, il a conduit à un repli des immigrés sur eux-mêmes et entraîné une 
faible "intégration » dans la société d'accueil, les immigrés se réunissant entre eux, n'entrant en relation avec la population française que dans le cadre du travail. Le temps servait à travailler et l'argent à économiser, ce qui implique qu'il n'y ait pas toujours eu un investissement dans l'apprentissage correct du français. Ainsi s'est développé un argot inventé fait d'hispanismes: chambra (chambre/habitación); pubela (poubelle/ basura); curra (cours/pasillo); retreta (retraite/jubilación); burones (bureaux/oficinas), etc. ${ }^{16}$. En résumé, le projet d'une migration de passage, temporaire, fonctionnelle, a supposé de passer le maximum de temps au travail, dans des conditions de vie difficiles. Ainsi, le poids des représentations sociales a-t-il conditionné la trajectoire de nos interlocutrices qui, motivées par la projection de la mobilité sociale en Espagne, ont bloqué leur ascension sociale à Paris.

« Nous avons tout investi en Espagne, au lieu d'investir ici, à Paris, cela a été une erreur que nous avons commise, parce que si au lieu d'investir dans des villages en Espagne, on l'avait fait ici, alors oui nous serions devenus les oncles d'Amérique. Parce que maintenant avoir un appartement à Paris, cela vaut beaucoup plus cher, c'est beaucoup mieux assuré qu'avoir un appartement dans un village en Espagne, là où nous avons investi " (Ana, qui migra avec son époux, concierge, XIe arrondissement de Paris).

«En Espagne, on a beaucoup évolué, les gens vivent mieux. Ils vivent mieux que nous, parce que nous n'avons pas voulu faire notre vie, parce que si nous avions voulu, nous aurions pu aller tous les jours au bar, avec ce que nous avons épargné. La différence, c'est que là-bas les personnes vivent au jour le jour. Là-bas, c'est chez eux et s'ils dépensent, et bien, ils dépensent chez eux! Nous, nous ne sommes pas venus pour dépenser. La majorité des émigrants ne dépensent pas, parce qu'on n'est pas venus pour ça, on est venus pour travailler et pour envoyer l'argent là-bas et ne pas le dépenser ici» (Leonor qui migra dans le cadre du regroupement familial, $\mathrm{XI}^{\mathrm{e}}$ arrondissement de Paris).

Dans l'espace social d'origine, la forte mobilité sociale des premières années de la migration se voit aussi bloquée. Le développement économique et social en Espagne fut tel, que la différence de revenus, qui apportait de grands avantages au migrant au début du cycle migratoire, se réduisit considérablement. Les migrants virent les intérêts de leurs comptes bancaires en Espagne sensiblement baisser. L'Espagne adhéra à la société de consommation de masse. La mentalité consumériste a remplacé l'accumulation monétaire et l'épargne, synonyme d'ascension sociale dans le monde rural. Le recours au crédit et au paiement échelonnés permet à la classe moyenne et ouvrière espagnole, d'accéder, de façon croissante, à des produits et des services auparavant destinés à quelques-uns. Dans ce nouvel espace social, la voiture et l'appartement du migrant ne sont plus des signes de richesse comme dans les années 1960. Au retour de vacances, certaines Espagnoles sont désorientées par les pratiques de consommation de la société espagnole. Les robes achetées dans les magasins parisiens "Tati», qui, dans les années 1960, provoquaient des ravages en Espagne, perdront de leur glamour dans les années 1990 devant les modes vestimentaires de la classe moyenne espagnole. La mobilité sociale du migrant, au-delà du capital économique accumulé, portera moins ses fruits dans l'espace social d'origine qui, en peu de temps, a connu une importante mobilité « collective».

«Les gens qui ont émigré ont amélioré leur niveau de vie économique. Au début de l'émigration, il y avait beaucoup d'amélioration, mais plus aujourd'hui. Celui qui est resté en Espagne a vécu beaucoup mieux que celui qui a émigré, mes sœurs vivent comme des princesses. Tout le monde a un ordinateur, une télévision, tous les enfants ont des bicyclettes... On remarque beaucoup de changements. L'Espagne a surgi de partout à partir de la fin des années soixante. Avant on remarquait la 
différence de ceux qui émigraient, parce que ceux qui venaient de l'extérieur s'étaient offert les premières voitures, ils savaient aussi comment s'habiller. Tu voyais les filles avec trois robes... À cette époque, cela se voyait, au début. Maintenant, cela ne se voit plus. Avant pour trouver quelque chose, il fallait sortir du pays, mais maintenant celui qui est resté au village, il a les mêmes choses et il vit mieux. Tous ceux qui ont émigré ont apporté beaucoup d'argent au village, ils donnaient de l'argent à ceux qui restaient, aux ouvriers, aux peintres. Ceux des bars également, ils ont fait des bénéfices quand les émigrés rentraient en vacances. Il y a des gens qui n'ont pas émigré, mais qui ont vécu avec l'argent envoyé d'ici » (María, pionnière de la migration familiale, $\mathrm{XVI}^{\mathrm{e}}$ arrondissement de Paris).

Les enfants des migrants ont amélioré leur sort. La majorité d'entre eux ont suivi des études secondaires ou supérieures parfois dans des filières technologiques et courtes (BTS), ou plus longues, optant pour les cursus universitaires. Les migrants espagnols se sont évertués à faire sortir leurs enfants des secteurs économiques dans lesquels ils étaient confinés. La maîtrise de la langue française et espagnole est également un acquis important pour les enfants de l'immigration, qu'ils peuvent valoriser sur le marché du travail. Cependant, certaines de nos interlocutrices n'ont pas le sentiment que les enfants des immigrés (excepté la maîtrise du français et l'acquisition d'une éducation en France, considérée de meilleure qualité qu'en Espagne) aient significativement amélioré leur sort comparativement à leurs neveux, et, plus généralement, aux enfants de la classe ouvrière en Espagne. En effet, l'accès massif des jeunes générations d'Espagnols à l'Université a permis à de nombreux enfants de poursuivre des études.

"Je ne crois pas que les enfants des émigrants aient amélioré leur niveau plus que ceux qui sont restés là-bas. Parce que maintenant en Espagne tout le monde fait des études, tout le monde donne une éducation à ses enfants. Moi, je trouve que mes neveux sont comme mes enfants, mes frères qui sont restés leur ont donné un niveau d'études comparable et maintenant, là-bas, ils sont comme nos enfants ici ou mieux » (Patricia, qui migra seule, mariée, femme de ménage, $\mathrm{XI}^{\mathrm{e}}$ arrondissement de Paris).

Non intégrées en Espagne, considérées comme des étrangères (dénommées «Françaises» dans leurs communautés d'origine), les femmes migrantes restées à Paris, évaluent souvent négativement leur expérience de vie: le sacrifice ne s'est pas traduit à long terme par une trajectoire spécialement ascendante, ni dans la société d'accueil, ni dans l'espace social d'origine. Les Espagnols de la classe ouvrière qui n'ont pas quitté le pays, les frères, les cousins, les voisins possèdent aujourd'hui un appartement, une voiture, ils sont bien vêtus, leurs pratiques de consommation et de loisirs sont satisfaisantes, leurs enfants ont également étudié... Si au départ, la migration a supposé une forte mobilité ascendante, actuellement, les migrants ne jouissent plus d'une position spécialement privilégiée dans la hiérarchie sociale d'origine. La logique de la consommation prime comme symbole de réussite sociale, tandis que la mentalité de l'épargne a perdu la bataille dans un pays de nouveaux riches. Des facteurs extérieurs à la décision de l'individu ont joué un mauvais tour aux migrants, en termes de stratégie de mobilité sociale. Cette stratégie d'ascension sociale, individualiste ou familiale, a été préjudiciable sur le long terme aux protagonistes de la migration, tandis qu'elle a profité collectivement à la société espagnole.

« Pour moi, venir ici à Paris, cela ne s'est pas bien passé. Maintenant, j'ai 60 ans et je n'ai rien et ma sœur qui est restée à Séville, elle a tout : une belle maison, un mari et des enfants. Son mari travaille dans l'aviation. Si je compare sa situation avec la mienne, j'ai honte de la façon dont je vis. Lorsqu'elle est venue me rendre visite à 
Paris, je n'ai pas voulu que ma sœur rentre chez moi » (Rosa, qui migra seule, mère célibataire, concierge, $\mathrm{XVI}^{\mathrm{e}}$ arrondissement de Paris).

"J'étais chez le coiffeur en Espagne et elles ont commencé à dire que maintenant il y avait la crise économique et elles disaient: "c'est la faute des émigrants qui n'apportent plus d'argent en Espagne". Moi, je me fais couper les cheveux en Espagne car le coiffeur ici c'est moins cher qu'à Paris, on regarde toutes nos dépenses, nous. La coiffeuse m'a dit que maintenant il y avait moins d'argent et que c'était la faute des émigrants : ils ont créé de mauvaises habitudes, ils sont venus avec leurs millions et maintenant c'est terminé. Moi, je dis que s'ils avaient vécu comme les autres, cela ne leur serait pas arrivé. Maintenant, l'argent n'entre plus en Espagne, parce que nous ne pouvons plus économiser, on ne peut plus en envoyer autant qu'avant. On a mis l'Espagne debout et maintenant on ne peut plus épargner. C'est triste qu'on rejette sur nous la faute du gaspillage. C'est chez moi et je me sens mal » (Teresa, mariée, concierge, $\mathrm{XI}^{\mathrm{e}}$ arrondissement de Paris).

On peut ainsi parler de paradoxe pour qualifier la situation des femmes espagnoles à Paris : il se résume au fait que tout en ayant axé leur vie professionnelle en France, ces femmes (et éventuellement leur conjoint) ont programmé une mobilité sociale en Espagne. Or, des déterminants d'ordre micro et macro structurels (position dans le cycle de vie, développement économique de l'Espagne) ont conditionné l'expérience de vie des migrants, de telle sorte que leur projet migratoire d'ascension sociale s'est traduit, dans certains cas, par une trajectoire de stagnation à long terme (voir figure 1).

Figure 1

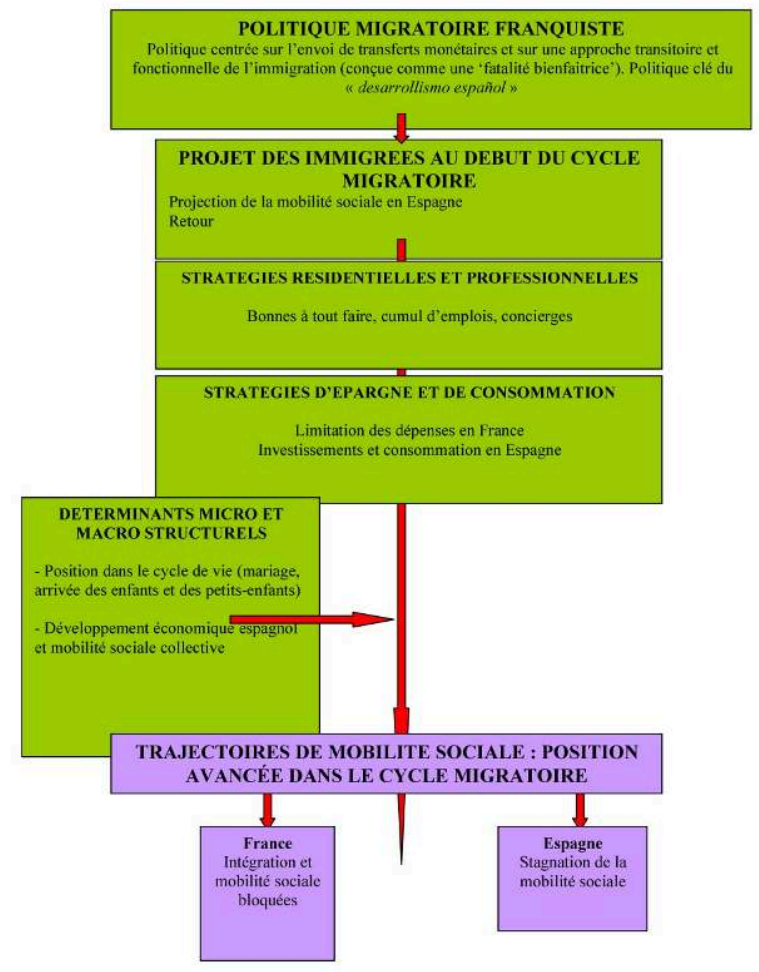

\section{Conclusion}

L'émigration, moteur du développement espagnol des années 1960-1970, s'est transformée sur le long terme en blocage de la mobilité sociale pour certains de ses 
protagonistes, qui ont vécu dans la logique du sacrifice et qui n'ont pas profité des fruits de leur travail. Ainsi, aux yeux de certaines de nos interlocutrices, ce sont les Espagnols qui n'ont pas migré qui ont réellement bénéficié de la stratégie de développement collectif. Les Espagnols ont pu profiter, au jour le jour, de la mobilité ascendante de l'ensemble de la société espagnole. Comme nous l'avons vu, l'instrumentalisation par le régime franquiste de l'émigration économique espagnole, qui a impulsé une mentalité cramponnée à l'idée d'épargner et de retourner dans son pays d'origine, fut une stratégie clé permettant d'assurer l'envoi de transferts monétaires des émigrés et, par conséquent, le « desarrollismo español » mais elle a eu un effet pervers sur ses protagonistes. Et ce développement économique impulsé par l'émigration a été, au long de l'histoire de l'Espagne, pensé au masculin, l'importance de la participation des femmes à ce processus n'ayant pas été prise en compte (bonnes à tout faire, femmes de ménage et concierges). Reléguées à Paris et cantonnées à la réalisation de travaux de reproduction sociale, leur participation économique a été occultée. Oubli de leur contribution au développement économique espagnol que cet article a voulu contribuer à réparer.

La réserve féminine armée de brosses, de coiffes et de serpillières a déjà atteint les villes et les campagnes espagnoles. Réserve de main-d'œuvre également destinée à couvrir les postes de travail les plus dévalorisés socialement. Femmes philippines, marocaines, dominicaines, péruviennes, équatoriennes, colombiennes à qui la société espagnole réserve, comme cela fut le cas avec les Espagnoles à Paris, des alcôves, des logements de service ou des appartements partagés. L'histoire se répète. Et nous trébuchons, à nouveau, sur la même pierre, puisque les gouvernements européens continuent à ne voir dans la migration qu'un problème de flux et de stocks, sans calculer le coût social des politiques migratoires et appréhender son caractère humain.

La recherche qualitative a permis de faire émerger les rêves, les stratégies et la reconstruction biographique des trajectoires de mobilité sociale des femmes espagnoles immigrées, bien que certaines lacunes ne soient pas comblées. En premier lieu, nous nous sommes interrogées sur la façon dont les femmes espagnoles à Paris jugent leur trajectoire de mobilité sociale, comparativement aux membres de leur famille, à leurs voisins, à leurs amis qui n'ont pas migré. Mais nous n'avons pas cherché à savoir ce que sont réellement devenus ceux qui sont restés en Espagne. Ont-ils eu plus de chance que ceux qui ont migré ? La réponse à cette question supposerait de réaliser un travail de terrain auprès de membres de la population restés en Espagne. Nous ne savons pas plus ce qu'il est advenu de ceux qui sont retournés en Espagne. Peut-être ceux qui ont su revenir à temps ont-ils pu profiter de l'élan qu'une émigration temporaire leur a donné, en accumulant une somme d'argent qui leur a permis d'investir en Espagne. Pour ces immigrants revenus à temps, la migration a-t-elle supposé une mobilité sociale ascendante en Espagne? De nouveau, la réponse à cette question suggère de mener un travail de terrain auprès de la population migrante rentrée en Espagne. 


\section{BIBLIOGRAPHIE}

ALONSO Luis Enrique et CONDE Fernando (1994) Historia del consumo en España : una aproximación a sus orígenes y primer desarrollo, Madrid, Debate.

ARAGON R. (1986) Panorama de la emigración española en Europa, Ministerio de Trabajo y Seguridad Social, Madrid.

ARONDO M. (1975) Moi, la bonne, Paris, Stock 2.

BARBIER R. (1983) Crónica amable de la caja de ahorros vizcaína, Bilbao, Caja de Ahorros Vizcaína.

BLANCO María (1982) Immigrées Espagnoles dans l’Agglomération Parisienne, Mémoire de Maîtrise, Université de Paris I.

BLAU P.M. and DUNCAN O.D. (1967) The American Occupational Structure, NY, Wiley.

CUIN C.H. (1993) Les sociologues et la mobilité sociale, Paris, PUF.

DREYFUS-ARMAND Geneviève et TEMIME Emile (1998) Les Espagnols en France au Xxe siècle, in L. Gervereau, P. Milza et E. Temime, Histoire de l'immigration en France au $\mathrm{xx}^{\mathrm{e}}$ siècle, Paris, Somogny, Éditions d'Art, pp. 67-76.

EWENCZYK Pierre (1981) Les Espagnols, in J-P. Garson et G. Tapinos Dir., L'argent des immigrés. Revenus, épargne et transferts de huit nationalités immigrées en France, INED, Travaux et Documents, Cahier no 94, PUF, pp. 71-98.

GARMENDIA Jose Antonio Éd. (1981) La emigración española en la encrucijada : marco general de la emigración de retorno, Madrid, CIS.

GRESLE François et al. (1994) Dictionnaire des sciences humaines, Paris, Nathan.

GUTIERREZ Héctor (1984) Les femmes espagnoles immigrées en France, Population, (39), mai-juin, pp. 617-620.

HERMET Guy (1967) Espagnols en France. Immigration et culture, Paris, Éditions Ouvrières.

LEONETTI Isabelle (1979) L'immigration féminine espagnole aujourd'hui, Migrants Formation, 32-33 (1), mars, pp. 17-148.

LILLO Natacha (2004) La Petite Espagne de la Plaine-Saint-Denis 1900-1980, Paris, Éditions Autrement. MARTINEZ Conchita (1986) Travailleurs espagnols à Paris : quartiers de résidence et mode de vie, Mémoire de DESS, Institut d'Urbanisme de l'Académie de Paris, Université de Paris VIII.

OSO Laura (2004) Españolas en París. Estrategias de ahorro y consumo en las migraciones internacionales, Barcelona, Bellaterra.

OSO Laura (2001) Domestiques, concierges et prostituées : migration et mobilité sociale des femmes immigrées, espagnoles à Paris, équatoriennes et colombiennes en Espagne, Thèse de Doctorat, IEDESUniversité de Paris I-Panthéon Sorbonne.

OSO Laura (2002) Stratégies de mobilité sociale des domestiques immigrées en Espagne, Revue Tiers Monde, XLIII (170), avril-juin, pp. 287-305.

PARRA LUNA Francisco (1981) La emigración española a Francia, 1962-1977, Madrid, Instituto Español de Emigración. 
REMBAUVILLE N.F. (1964) Guide bilingue ménager à l'usage des employées de maison espagnoles et de leurs employeurs, Paris, Presses de la Cité, Librairie Polytechnique Béranger.

RUBIO Javier (1974) La emigración española a Francia, Barcelona, Ariel.

TABOADA LEONETTI Isabelle (en collaboration avec Michèle GUILLON) (1987) Les immigrés des beaux quartiers. La communauté espagnole dans le XVIe, Paris, CIEMI/L'Harmattan.

TRIBALAT Michèle et al. (1996) De l'immigration à l'assimilation : enquête sur les populations d'origine étrangère en France, Paris, La Découverte.

TOUR Bruno (2003) De Valence à Paris : Itinéraires des « bonnes » espagnoles (1940-1974), Mémoire de maîtrise d'histoire, Paris, Université de Paris VII.

\section{NOTES}

1. Federación de Asociaciones y Centros de Españoles Emigrantes en Francia.

2. Si la thématique de l'immigration espagnole dans les années 1960-1970 a suscité une production scientifique - parmi laquelle il convient de citer les travaux de Rubio (1974), Parra Luna (1981) et Hermet (1967) - , peu de recherches relatives au rôle des femmes employées, domestiques et concierges ont cependant été publiées. Parmi ces dernières se détachent celles de Taboada Leonetti (Taboada-Leonetti, 1987; Leonetti, 1979). On peut encore trouver quelques mémoires de DEA, DESS ou maîtrise qui traitent, de façon plus ou moins directe, de ce thème (Blanco, 1982 ; Martínez, 1986 ; Tour, 2003).

3. Cependant, la méthode biographique entraîne une série d'inconvénients dérivés du souvenir lointain des motivations et des projets migratoires initiaux, de telle sorte que parfois la vision des projets migratoires s'estompe au fur et à mesure que le temps passe. Nous sommes face à une réflexion sur la notion de bilan de vie et sur la manière dont la mémoire est recomposée à la lumière du présent.

4. Sur les différentes vagues de l'immigration espagnole en France, voir Dreyfus-Armand et Temime (1998).

5. La migration espagnole installée à la Plaine-Saint-Denis, qui date du début du $\mathrm{xx}^{\mathrm{e}}$ siècle, présente, par exemple, selon Natacha Lillo, une forte composante familiale et ce, à l'inverse de ce qu'il est possible de rencontrer parmi la migration économique espagnole à Paris (années 1960-1970) où l'on trouve de nombreuses femmes seules.

6. En témoigne par exemple, la publication d'un guide bilingue destiné aux employeurs français et aux employées de maison espagnoles (Rembauville, 1964).

7. Cette recherche s'inscrit dans le cadre d'une thèse doctorale soutenue à l'IEDES (Université de Paris I-Sorbonne) et dirigée par M. Bruno Lautier (Oso, 2002). Elle a pu être réalisée grâce à une bourse octroyée par le Ministère espagnol de l'Éducation et de la Culture (Programa de Becas de Formación de Personal Investigador en el Extranjero), ce qui m'a permis de résider à Paris afin d'effectuer le travail de terrain (1998-1999). Je remercie cette institution pour l'aide financière accordée, ainsi que Pilar Soler, le Père Sindo, la Sœur Soledad, Mercedes Romero, la Maison d'Espagne et la FACEEF pour l'aide qu'ils m'ont fournie lors du travail de terrain. En dernier lieu, je remercie Michelle Guillon, Mirjana Morokvasic et Christine Catarino pour leurs critiques constructives ayant permis d'améliorer ce texte. Afin de préserver l'anonymat des personnes interviewées, les noms apparaissant tout au long de ce texte sont des pseudonymes. Les résultats de cette recherche ont été publiés récemment en Espagne (Oso, 2004).

8. Nous avons réalisé, au total, cinquante-deux entretiens en profondeur parmi lesquels 44 étaient individuels et huit en groupe (quatre avec deux participantes, deux avec trois participantes et deux réunions de groupe avec quatre participantes chacune). À ceci, il faut 
ajouter les contacts informels noués pendant l'observation réalisée au cours de différents événements auxquels nous avons assistés. Parmi l'ensemble des personnes contactées pour les entretiens, 57 étaient des femmes et 9 des hommes. La plupart des personnes contactées étaient des immigrés espagnols à Paris (56, dont 49 étaient des femmes). Dix d'entre elles étaient des informateurs clé en contact avec la réalité étudiée.

9. Barbier, 1983, cité par Alonso et Conde, $1994: 66$.

10. En 1958, elle devint membre du Fonds Monétaire International et de la Banque Mondiale. En 1959, elle adhéra à l'OCDE.

11. Approximativement « développement espagnol ».

12. Des travaux de recherche ont déjà analysé le rôle des transferts monétaires ainsi que de l'épargne des immigrés espagnols en France, citons par exemple l'étude réalisée par Pierre Ewenczyk (1981). Cependant, à la différence de ce travail, à caractère essentiellement quantitatif, notre approche, qualitative, vise à appréhender les stratégies et représentations de consommation et d'épargne, ainsi que les pratiques quotidiennes des immigrées comme partie intégrante d'une stratégie, plus ample, de mobilité sociale.

13. Notons que diverses pratiques sociales mentionnées (économies drastiques, récupération d'objets dans les poubelles, mise au travail des enfants, présentation de soi dans une position désavantageuse afin d'obtenir des avantages matériels) étant socialement désapprouvées, elles sont le plus souvent rapportées par nos interviewées en mettant en scène des personnes connues d'elles et non à la première personne.

14. Cependant, actuellement, l'amélioration des communications et les facilités de locomotion font que beaucoup de familles des migrants espagnols découvrent leurs véritables conditions de vie à Paris.

15. Sur le retour des immigrés espagnols, voir Garmendia (1981).

16. Un autre indicateur du repli sur elle-même de l'immigration étudiée est le fait que la grande majorité des mariages eurent lieu entre compatriotes. L'exploitation par l'auteur des registres paroissiaux de l'Eglise de la Pompe montre que dans les années 1963 et 1973, respectivement $73 \%$ et $68 \%$ des mariages contractés et enregistrés l'ont été entre Espagnols (Oso, 2004). Comme nous le voyons, les données de notre travail de terrain mettent en cause l'image d'une immigration espagnole comme communauté intégrée à la société française.

\section{RÉSUMÉS}

Cet article cherche à sortir de l'oubli la migration des femmes espagnoles arrivées à Paris dans les années 1960/1970 pour travailler comme employées domestiques ou concierges. L'approche théorique est centrée sur l'analyse des représentations, des stratégies et des trajectoires de mobilité sociale des femmes immigrées (professionnelles, résidentielles, d'épargne et de consommation). La recherche est basée sur des entretiens en profondeur et des histoires de vie. Elle remet en question la perspective du migrant rationnel qui calcule son ascension sociale dans le cadre d'une société ouverte. La mobilité sociale des individus est subordonnée à des déterminants structurels, et dépend des stratégies d'autres acteurs sociaux avec lesquelles leurs propres stratégies peuvent entrer en contradiction. Ainsi, les femmes immigrées, qui s'insèrent dans deux types d'espaces sociaux (d'origine et d'accueil), peuvent voir leur trajectoire de mobilité sociale bloquée, tandis que leurs stratégies individuelles ou familiales de mobilité sociale 
ont constitué un facteur clé du développement économique espagnol des années soixante, bénéficiant collectivement à la société espagnole.

Paradoxical Success of Spanish Domestic Workers in Paris: Social Mobility Strategies and Life Trajectories

This article highlights the migration of Spanish women who arrived to Paris in the sixties and the seventies to be employed as domestic workers and concierges (apartment building caretaker). The theoretical approach focuses on the analysis of the representations, strategies (occupational, residential, consumer and saving) and trajectories of social mobility among female immigrants. It is based on in depth interviews and life histories. The evidence from my study suggests that social mobility is far removed from the perspective of the rational migrant who calculates his/ her social promotion within the framework of an open society. Instead, social mobility is determined by structural factors and by often contradictory strategies used by other social actors. Paradoxically, whereas the individual and family mobility strategies of migrant women have been a key factor in Spanish economic development of the sixties, improving collective social mobility of Spanish society, their own upward mobility has meanwhile stagnated.

El ascenso social paradójico de las criadas españolas en París : estrategias de movilidad social y trayectorias biográficas

Este artículo busca sacar del olvido la migración de mujeres españolas que llegaron a París durante los años 1960 / 1970 para trabajar como empleadas domésticas o porteras. La perspectiva teórica de la investigación se centra en el análisis de las representaciones, estrategias y trayectorias de movilidad social de las mujeres inmigrantes (profesionales, residenciales, de ahorro y consumo). La metodología utilizada ha sido cualitativa (entrevistas en profundidad e historias de vida). La investigación cuestiona la perspectiva del migrante racional que calcula su ascenso social en el marco de una sociedad abierta. La movilidad social está condicionada por determinantes estructurales, así como por las estrategias de otros actores sociales, pudiendo entrar en contradicción. De esta manera, las mujeres inmigrantes, que se encuentran sumergidas en dos espacios sociales (de origen y de acogida), han podido ver bloqueada su trayectoria de ascenso social, mientras que sus estrategias individuales o familiares de movilidad social han constituido un factor clave del «desarrollismo español» de los años sesenta, beneficiando colectivamente a la sociedad española.

INDEX

Mots-clés : personnel de service, femmes, mobilité sociale, épargne, relations avec le pays d'origine, Espagnols

Index géographique : Paris

\section{AUTEUR}

\section{LAURA OSO CASAS}

Profesora Titular de Universidad, Facultade de Socioloxia, Universidade da Coruña, Campus Elviña s/n, 15071- A Coruña, Espagne, osofac@udc.es 